\title{
Sistema misto de madeira e concreto: uma abordagem numérico e experimental para altas temperaturas
}

\author{
Timber and concrete composite system: a \\ numerical and experimental approach for \\ high temperatures
}

\footnotetext{
${ }^{1}$ Universidade Paulista "Júlio de Mesquita Filho" - UNESP, Itapeva, SP, Brasil. e-mail: molina@itapeva.unesp.br

${ }^{2}$ Escola de Engenharia de São Carlos - EESC/USP, São Carlos, SP, Brasil. e-mail: calil@sc.usp.br
}

\section{RESUMO}

Este trabalho teve a finalidade de avaliar o perfil de temperatura em duas vigas mista de madeira e concreto com seção transversal $\mathrm{T}$ e sistema de conexão metálico submetida à altas temperaturas. $\mathrm{O}$ sistema de conexão, neste caso, foi efetuado por barras de aço comum, com 12,5 mm de diâmetro, igualmente espaçadas ao longo da interface madeira-concreto. A seção transversal $\mathrm{T}$ da viga foi composta por uma alma de madeira com dimensões $5 \mathrm{~cm}$ x $15 \mathrm{~cm}$ e mesa de concreto com $30 \mathrm{~cm}$ x $5 \mathrm{~cm}$. Foi considerado para as vigas mistas um tempo de exposição ao fogo de 40 minutos. A simulação numérica foi realizada a partir da utilização do software ANSYS [1], versão 10.0. Os resultados das simulações numéricas foram comparados com os resultados experimentais de temperatura obtidos para as duas vigas mistas, as quais foram instrumentadas com termopares e submetidas à altas temperaturas em um forno horizontal de ensaios construído na EESC/USP para tal finalidade. Os resultados numéricos e experimentais apresentaram uma boa concordância neste caso. Como resultado, são apresentados os perfis de temperatura na seção transversal para vários tempos de aquecimento, assim como toda estratégia de modelagem numérica e os elementos finitos utilizados na determinação dos perfis de temperatura.

Palavras-chave: simulação numérica, incêndio, viga mista de madeira e concreto, análise experimental.

\section{ABSTRACT}

The aim of this work was the evaluation of the temperature profile in two composite timber and concrete beam with cross-section $\mathrm{T}$ and metallic connection system subjected to high temperatures. The connection system, in this case, was performed by use of steel bars with $12.5 \mathrm{~mm}$ diameter, equally spaced, along the concrete-timber interface. The cross section $\mathrm{T}$ of the beam consisted of a timber piece with dimensions $5 \mathrm{~cm}$ x $15 \mathrm{~cm}$ connected of a concrete slab with $30 \mathrm{~cm}$ x $5 \mathrm{~cm}$. To the composite beams, a total time of fire exposure equal to 40 minutes was considered. The numerical modeling of the system was carried out using the ANSYS software [1], version 10.0. The results of numerical simulations were compared with the experimental results of temperature obtained by the two composite beam, which were instrumented with thermocouples and subjected to high temperature in a horizontal furnace constructed on EESC/USP for this purpose. The numerical and experimental results showed good agreement in this case. As a result, the temperature profiles are shown in cross section for various heating times and all numerical modeling strategy and the finite element used to determine the temperature profiles.

Keywords: numerical simulation, fire, timber and concrete composite beam, experimental analysis.

\section{INTRODUÇÃO}


Atualmente, no Brasil, existe a necessidade do desenvolvimento de pesquisas voltadas para a análise de elementos estruturais em situação de incêndio, seja em caráter teórico, numérico ou experimental. A simulação das condições de incêndio de maneira mais realista consiste em um requisito importante para a análise do projeto de edificações. O sistema estrutural misto de madeira e concreto é apresentado como uma alternativa que pode aumentar a vida útil da madeira em diversas aplicações estruturais. A utilização de concreto no sistema misto protege a madeira principalmente contra os desgastes por abrasão e, a utilização de madeira tratada com CCA, neste caso, garante uma vida útil para a madeira de mais de 25 anos contra apenas 5 anos de viga quando se considera a madeira sem tratamento. Além disso, o potencial de integração da madeira com tabuleiros de pontes e pisos permite a obtenção de um sistema com maior resistência e rigidez. No entanto, existe a necessidade da utilização de um sistema de ligação metálico entre a madeira e o concreto para que os referidos materiais trabalhem em conjunto na flexão.

Os sistemas mistos de madeira e concreto são de fácil implementação e apresentam vantagens econômicas e estruturais com relação à madeira e ao concreto nas suas formas simples. Investigações experimentais do comportamento do sistema misto madeira-concreto em altas temperaturas foram realizadas na Nova Zelândia por [2] e na Suíça por [3] para avaliação da resistência e rigidez do sistema. Nos testes realizados por [2], por exemplo, foram analisados, em altas temperaturas, pisos mistos formados por vigas de LVL ( $L a$ minated Venner Lumber) espaçadas de $20 \mathrm{~cm}$ com laje de concreto de resistência igual a $30 \mathrm{MPa}$ e $65 \mathrm{~mm}$ de espessura. Como resultado, desse estudo observou-se a diminuição das seções transversais das vigas de LVL pela queima e a tendência de expansão térmica do concreto que foi restringida pela ação conjunta do sistema de conexão metálico usado entre a madeira e o concreto. Na Suíça, os estudos desenvolvidos por [3] envolveram a utilização de um modelo analítico calibrado a partir de coeficientes de modificação para as propriedades dos materiais de lajes mistas de madeira e concreto, em altas temperaturas. Os resultados do modelo foram comparados, neste caso, com os resultados experimentais obtidos para a estrutura em escala real e estes mostraram boa aproximação. Trabalhos experimentais sobre a madeira em condições de incêndio também foram desenvolvidos nos EUA por [4] tendo como foco principal a avaliação do tempo de incêndio de vigas de LVL em forno horizontal, concluindo que os referidos elementos de madeira ensaiados quando sujeitos às altas temperaturas resistem aos tempos de incêndio propostos em recomendações normativas do pais.

No Brasil, os estudos desenvolvidos por [5], envolvendo madeiras de eucalipto em altas temperatura, buscaram avaliar a espessura da camada de carbonização de vigas retangulares para diferentes tempos de incêndio. Por outro lado, no Brasil, a maioria dos estudos envolvendo elementos estruturais em altas temperaturas tinha até pouco tempo um caráter essencialmente numérico e envolviam os materiais aço e concreto e os sistemas mistos de aço e concreto [6, 7]. Estes estudos, desenvolvidos até no início do ano de 2012, tiveram um caráter essencialmente numérico por não existir no país até o então, equipamentos que permitissem a realização de testes de elementos estruturais com dimensões reais sob carregamento.

O primeiro forno horizontal do Brasil desta natureza entrou em funcionamento no início do segundo semestre de 2012, como relatado em [8]. Vale mencionar também que os custos para o ensaio de um elemento estrutural em escala real, e em altas temperaturas, podem chegar a valores em torno de 30 mil dólares [9]. Além disso, os modelos de cálculo para altas temperaturas baseiam-se em métodos numéricos tais como Método das Diferenças Finitas (MDF) e Método dos Elementos Finitos (MEF). Esses métodos permitem a obtenção da temperatura durante o processo de aquecimento. Para utilização destes métodos é necessária a utilização de programas computacionais com configurações e ferramentas compatíveis com o esforço numérico para a resolução do problema. Alguns programas utilizados, neste caso, são ADAPTIC, SUPRTEMPCALC, SAFIR e VULCANO. A análise numérica de transferência de calor também pode ser realizada por pacotes comerciais que tem como base o Método dos Elementos Finitos, tais como ANSYS, ABAQUS, ADINA e DIANA, todos conhecidas mundialmente. A análise térmica geralmente tem um caráter transiente, considerando o fato de que a temperatura num ambiente em chamas tem variação com o tempo.

Sendo assim, este trabalho foi desenvolvido pela necessidade de obtenção de informações sobre o comportamento de vigas mistas de madeira e concreto em altas temperaturas. A análise numérica aqui desenvolvida serviu, neste caso, como complementação para o estudo experimental realizado no forno horizontal construído no Laboratório de Estruturas da Escola de Engenharia de São Carlos da Universidade de São Paulo. O referido estudo considerou vigas mistas de madeira e concreto de seção transversal $\mathrm{T}$ com conectores formados por barras de aço verticais dispostos na interface dos materiais madeira e concreto. Como resultado, são apresentados os principais elementos finitos dos modelos numéricos, assim como toda a estratégia de modelagem numérica utilizada para as vigas mistas que foram ensaiadas experimentalmente. São também apresentados os campos de temperatura obtidos para as regiões dos conectores de cisalhamento e para toda a seção transversal T das vigas. São apresentadas ainda as principais propriedades térmicas dos materiais ma- 
deira e concreto e aço. Os detalhes dos ensaios experimentais realizados, assim como da instrumentação dos elementos submetidos às altas temperaturas também são apresentados.

\section{METODOLOGIA}

\subsection{Programa Experimental}

\subsubsection{Primeiro ensaio em altas temperaturas}

No primeiro teste do forno horizontal, considerou-se uma viga mista de madeira e concreto de seção transversal T com 6 metros de comprimento e conectores de aço CA-50 colados com resina epóxi (Compound 32), espaçados entre si de $16 \mathrm{~cm}$. Considerou-se madeira de Eucalyptus citriodora na umidade 16,93\%, com resistência a compressão de 82,13 MPa, módulo de elasticidade na compressão igual a $19727 \mathrm{MPa}$ e densidade de $1050 \mathrm{~kg} / \mathrm{m}^{3}$. Para o concreto considerou-se traço 1:2:2 em peso, com relação água-cimento $(\mathrm{a} / \mathrm{c}=0,42)$, resistência na compressão de 26,0 MPa e módulo de elasticidade na compressão de $24157 \mathrm{MPa}$. A distância considerada para o vão entre apoios, nesse caso, foi de 5,10 m. A seção transversal da peça de madeira serrada foi admitida com dimensões $5 \mathrm{~cm}$ x $15 \mathrm{~cm}$ e mesa armada de concreto com seção retangular de $30 \mathrm{~cm} \times 5$ $\mathrm{cm}$. A Figura 1 ilustra a configuração da viga e carregamento considerados na análise experimental. O carregamento em temperatura ambiente, assim como o carregamento em altas temperaturas foi aplicado nos terços do vão, sendo que o carregamento admitido no ensaio em altas temperaturas foi tomado com $30 \%$ do valor de ruptura obtido no ensaio em temperatura ambiente. A leitura das temperaturas na viga mista foi feita com a utilização de termopares dispostos em 14 pontos da seção transversal T, Figura 3(a). Esta primeira viga ensaiada em altas temperaturas teve a superfície superior da mesa de concreto revestida com manta refratária para evitar a transferência de calor entre os gases quentes do forno e a referida superfície. A instrumentação para colocação dos termopares do tipo K é mostrada na Figura 2. A Figura 3 mostra a configuração da seção transversal da viga mista usada para os ensaios em temperatura ambiente e também em altas temperaturas. $\mathrm{O}$ ensaio da viga em altas temperaturas no forno horizontal teve aquecimento controlado por uma curva padronizada de temperatura versus tempo [10]. Os deslocamentos no meio do vão das vigas (flechas) foram medidos por transdutores de deslocamentos posicionados do lado externo do forno de ensaios.

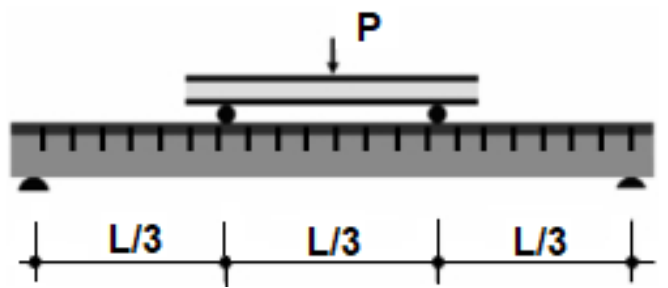

Figura 1: Configuração adotada para os ensaios em temperatura ambiente e em altas temperaturas das vigas mistas. 


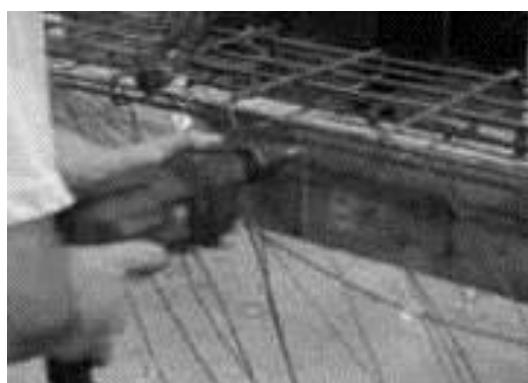

(a)

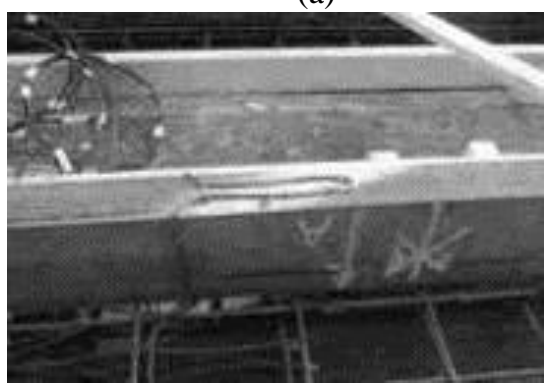

(c)

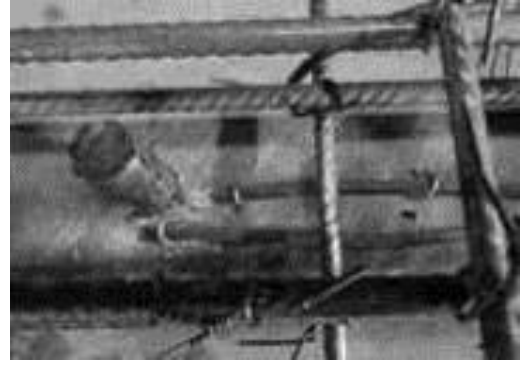

(b)

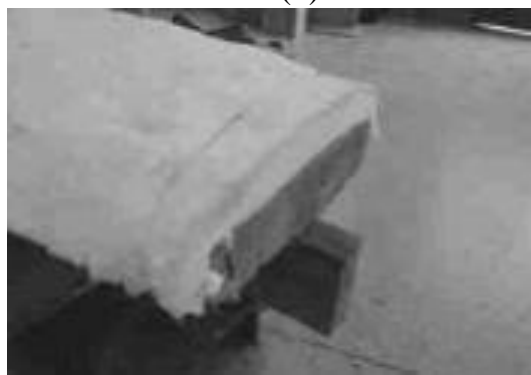

(d)

Figura 2: Instrumentação: (a) Furação; (b) Conector com termopares (c) Termopares na armadura; (d) Manta refratária.

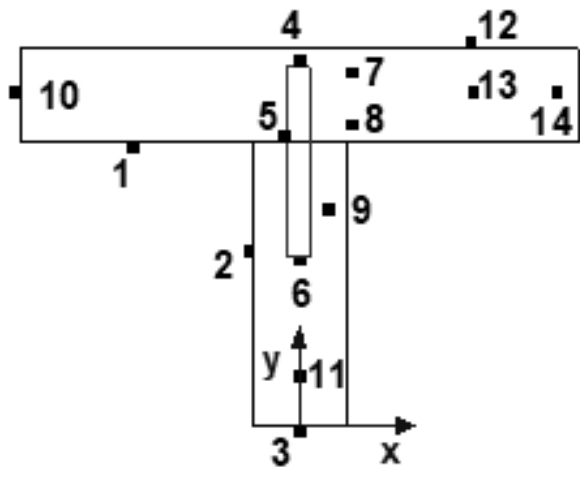

(a)

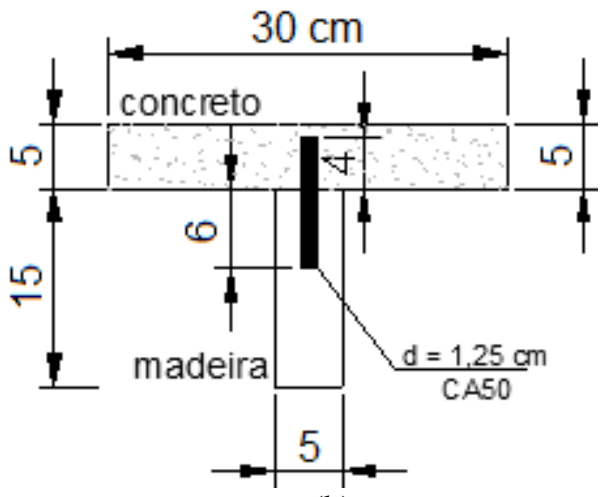

(b)

Figura 3: Seção transversal da viga mista: (a) Pontos de leitura das temperaturas; (b) Dimensões.

A Figura 4 mostra as etapas de instrumentação das vigas no interior do forno horizontal e os detalhes do ensaio propriamente dito. O forno utilizado, neste caso, atinge uma temperatura máxima de $1260{ }^{\circ} \mathrm{C}$. Segundo [9] não existem muitos tipos de strain gages para altas temperaturas e, devido à alta taxa de aquecimento inicial dos fornos, os poucos que existem fornecem leituras confiáveis até 20 minutos de exposição ao fogo, e apresentam custo relativamente elevados. No entanto, informações sobre as deformações dos elementos em altas temperaturas são importantes para a validação de modelos numéricos.

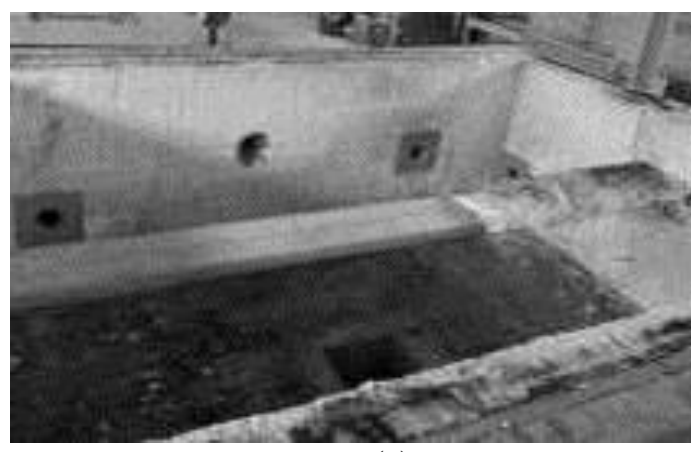

(a)

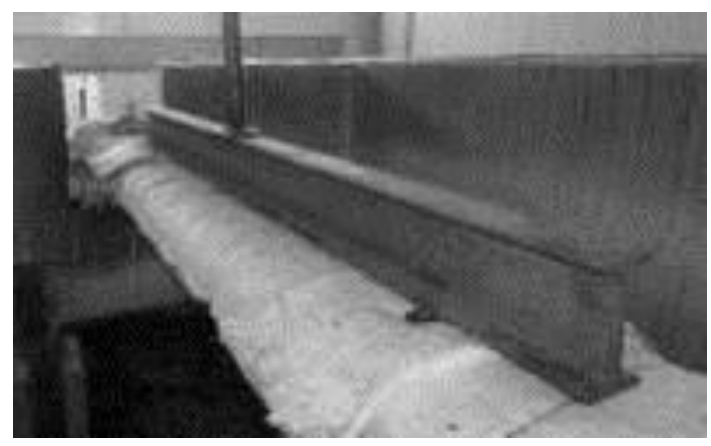

(b) 


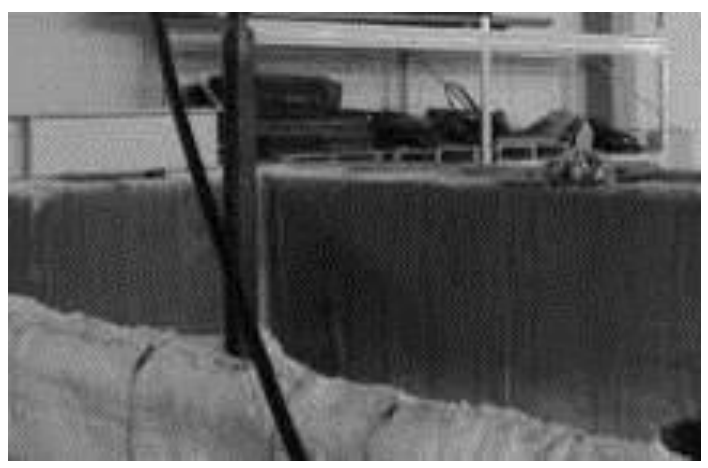

(c)

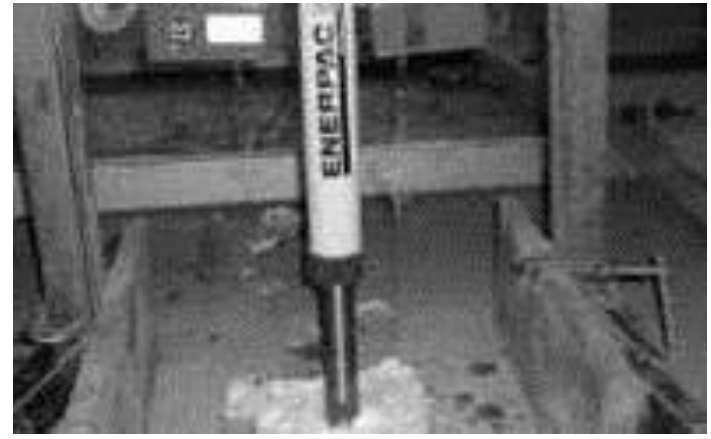

(d)

Figura 4: Detalhes do ensaio: (a) Viga posicionada; (b) Prova de carga com viga I; (c) Revestimento da viga I com manta refratária; (d) Revestimento do pistão de carga e transdutores de deslocamento posicionados externamente ao forno.

\subsubsection{Controle de parada do ensaio em altas temperaturas}

$\mathrm{O}$ ensaio da primeira viga mista em altas temperaturas foi conduzido com os seguintes critérios de parada do ensaio [10].

- Limite de deflexão, em mm (Equação 1):

$$
D=\frac{L^{2}}{400 \cdot d}
$$

- Taxa de deflexão em mm/min., Equação 2):

$$
\frac{d D}{d t}=\frac{L^{2}}{9000 \cdot d}
$$

Onde: L é vão livre considerado para a viga mista ( $\mathrm{mm})$ e d a distância entre as fibras externas das zonas comprimida e tracionada (altura da viga, $\mathrm{mm}$ ).

A falha da viga no ensaio de flexão em altas temperaturas só é atingida se ambos os critérios descritos nas Equações (1) e (2) forem verificados. O critério descrito na Equação (2) não deve ser aplicado até que o limite L/30 seja excedido, entendendo-se, neste caso, que o sistema de aplicação de carga não está mais aplicando carga devido ao aumento rápido da deflexão.

\subsubsection{Segundo ensaio em altas temperaturas}

No segundo ensaio realizado considerou-se uma viga mista de madeira e concreto com dimensões e propriedades idênticas àquelas apresentadas para a viga do $1^{\circ}$ ensaio. Porém a fixação dos conectores de cisalhamento foi feita, neste caso, sem a utilização de resina epóxi, ou seja, os conectores de cisalhamento foram fixados somente por pré-furação da madeira. Além disso, ao invés de um carregamento externo aplicado nos terços do vão considerou-se somente o peso próprio da viga e também não foi utilizada manta refratária na superfície superior do concreto durante a realização do ensaio em altas temperaturas.

\subsection{Programa numérico}

\subsubsection{Elementos pré-definidos do software ANSYS}

O modelo numérico desenvolvido neste trabalho foi feito com a base em elementos pré-definidos disponíveis na biblioteca interna do software [1], versão 10.0. A escolha de cada elemento finito foi feita a partir da consideração da sua representação sobre o comportamento a ser simulado. Na calibração do modelo numérico foram considerados os resultados numéricos e experimentais de outros pesquisadores obtidos para a madeira [11] como também para o concreto e aço $[12,13,14]$. A seção transversal da viga mista foi modelada a partir de elementos bidimensionais do tipo de plano (PLANE77) e do tipo superfície (SURF 151). Esses elementos foram utilizados para a implementação das condições de contorno de convecção e radiação. O elemento PLANE77 apresenta oito nós e um grau de liberdade por nó, que é a temperatura. O elemento SURF 151 apresenta dois nós e considera um nó adicional (Nó extra) localizado fora da malha de elementos finitos para 
o controle da temperatura de todos os nós da malha. Os elementos PLANE77 e SURF151 são mostrados nas Figuras 5 e 6, respectivamente.

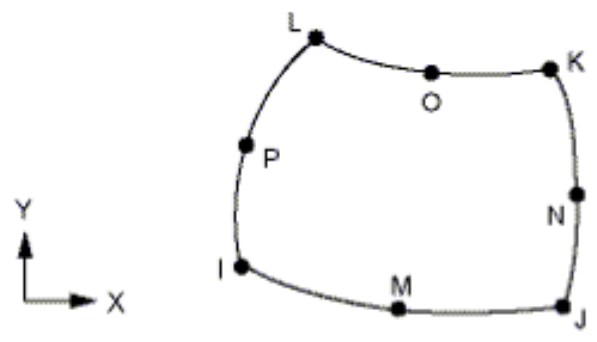

Figura 5: Elemento finito PLANE77 com um total de 8 nós. Fonte: [1].

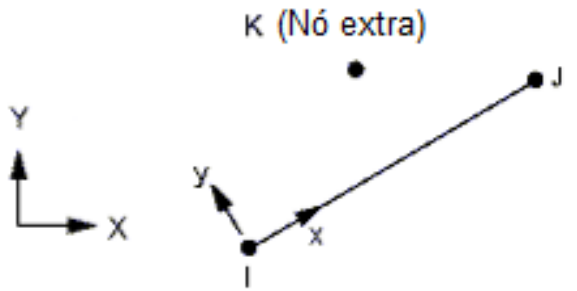

Figura 6: Elemento finito SURF151. Fonte: [1].

\subsubsection{Malha de elementos finitos}

A malha do modelo numérico construída a partir de [1] foi analisada a partir de diferentes níveis de refinamento até que os resultados conduzissem a respostas satisfatórias em termos de processamento da estrutura. O refinamento da malha, em cada uma das direções x e y (eixos de coordenadas do ANSYS) no plano, foi definido por 7 principais parâmetros. Na Figura 7 os parâmetros relacionados em (a), (b) e (c) são referentes às discretizações das peças de concreto, aço e madeira, respectivamente. A Tabela 1 apresenta três diferentes configurações de malhas avaliadas para o modelo numérico de viga mista com conectores verticais. Os valores seguidos do símbolo (c) referem-se às peças de concreto, seguidos do símbolo (a) referem-se ao conector de aço e os valores acompanhados do símbolo (m) são referentes às peças de madeira.

Tabela 1: Discretizações de malhas testadas para a viga mista com conectores verticais.

\begin{tabular}{c|c|c|c|c|c|c|c}
\hline \multirow{2}{*}{ MALHAS } & \multicolumn{7}{|c}{ PARÂMETROS DA MALHA } \\
\cline { 2 - 8 } & $\mathrm{nx}$ & $\mathrm{nx}, 1$ & $\mathrm{nx}, 2$ & $\mathrm{ny}$ & $\mathrm{ny}, 1$ & $\mathrm{ny}, 2$ & $\mathrm{ny}, 3$ \\
\hline $\mathrm{M} 1$ & $14(\mathrm{c})$ & $5(\mathrm{c})$ & $1(\mathrm{c})$ & $9(\mathrm{c})$ & $6(\mathrm{c})$ & $10(\mathrm{a})$ & $5(\mathrm{c})$ \\
\hline M2 & $56(\mathrm{c})$ & $5(\mathrm{c})$ & $1(\mathrm{c})$ & $36(\mathrm{c})$ & $24(\mathrm{c})$ & $40(\mathrm{a})$ & $20(\mathrm{c})$ \\
\hline M3 & $28(\mathrm{c})$ & $5(\mathrm{c})$ & $1(\mathrm{c})$ & $18(\mathrm{c})$ & $12(\mathrm{c})$ & $20(\mathrm{a})$ & $10(\mathrm{c})$ \\
\hline
\end{tabular}




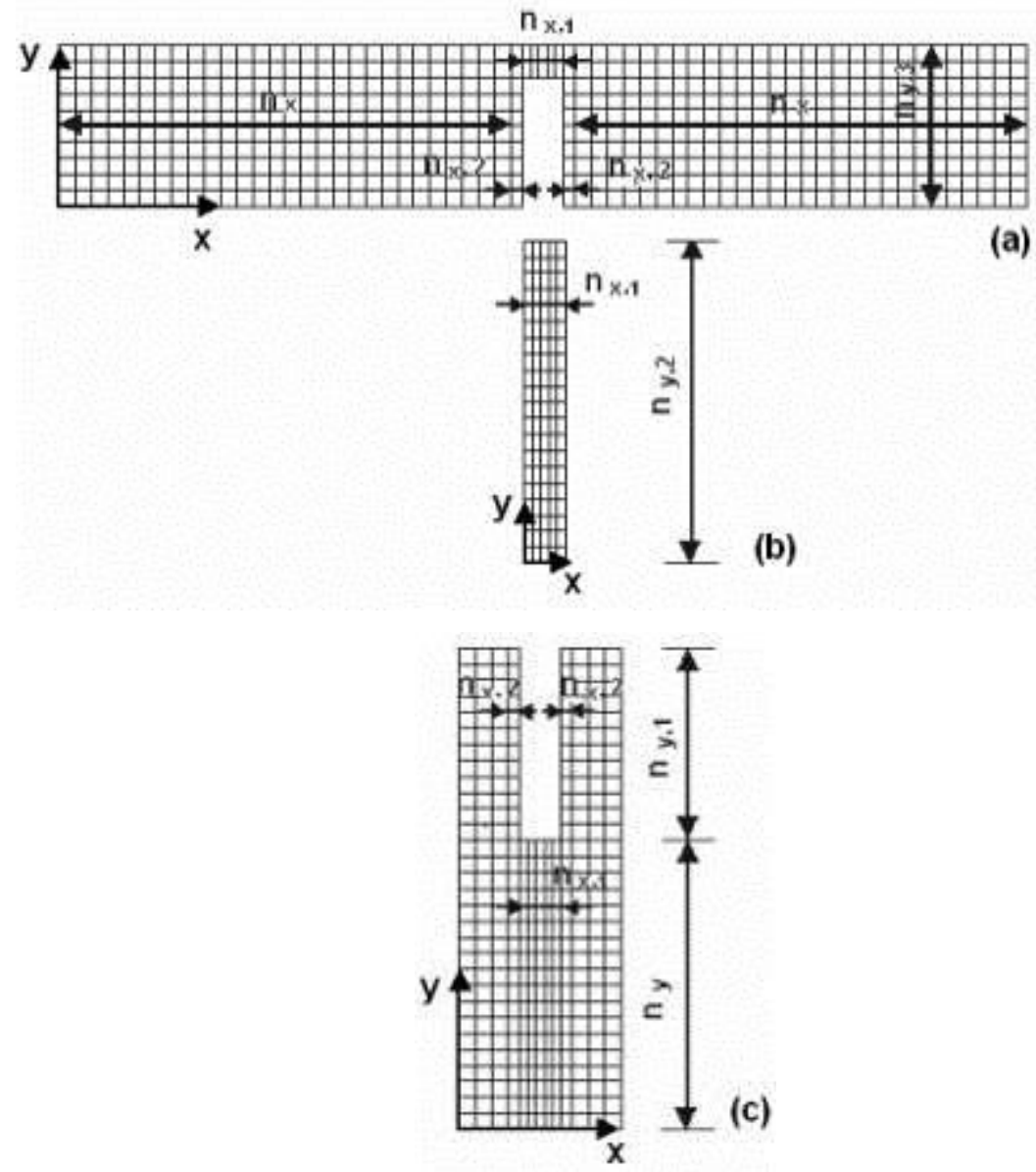

Figura 7: Parâmetros utilizados na definição da malha de elementos finitos da seção transversal da viga mista.

Onde: $\mathrm{n}_{\mathrm{x}}$ é o número de elementos na direção da largura da seção referente a mesa de concreto; $\mathrm{n}_{\mathrm{x}, 1} \mathrm{o}$ número de elementos na direção da largura da seção mista na região do conector, referente a mesa de concreto, alma de madeira e conector de aço; $\mathrm{n}_{\mathrm{x}, 2} \mathrm{o}$ número de elementos na direção da largura da seção mista nas proximidades da região do conector, referente a mesa de concreto e alma de madeira; $\mathrm{n}_{\mathrm{y}}$ o número de elementos na direção da altura da seção mista, referente a alma de madeira; $\mathrm{n}_{\mathrm{y}, 1}$ o número de elementos na direção da altura da seção referente a alma de madeira, na região do conector; $\mathrm{n}_{\mathrm{y}, 2} \mathrm{o}$ número de elementos na direção da altura da seção mista, referente ao conector de aço e $\mathrm{n}_{\mathrm{y}, 3} \mathrm{o}$ número de elementos na direção da altura da seção mista referente a mesa de concreto.

Os resultados das temperaturas nos pontos indicados na Figura 3(a) considerando-se as configurações M2 e M3 foram um pouco melhores que os resultados obtidos para a configuração M1, que foi menos discretizada, quando comparados com os valores experimentais. Observou-se, no entanto, uma maior estabilidade numérica para a configuração M3, sendo que os resultados obtidos para as configurações M2 e M3 foram praticamente os mesmos. No entanto, o tempo de processamento para a configuração M2 foi duas vezes maior que o tempo de processamento da configuração M3. Por essas razões, optou-se pela utilização da configuração M3 na modelagem da seção de viga mista com conectores verticais. Assim, a malha de elementos finitos da seção transversal da viga mista foi discretizada no modelo em elementos do tipo plano (PLANE77) totalizando 1020 elementos finitos na seção transversal como mostrado. Desse total, 590 elementos finitos compõem a parte do concreto, 330 a parte da madeira e 100 da parte do aço.

\subsubsection{Superfícies de aquecimento}

Para o aquecimento padronizado da seção de acordo com [10] foi necessária a definição de superfícies de controle para o modelo. Essas superfícies foram necessárias para a consideração dos efeitos de radiação e convecção. As superfícies foram acopladas a um nó externo à malha (Nó extra) que foi utilizado para a aplicação da temperatura externa ao modelo. Essas superfícies foram utilizadas para a simulação de um incêndio com aquecimento em três faces, conforme mostra a Figura 8. Posteriormente, foi definida uma outra superfície de convecção (com elemento do tipo SURF 151) na face superior e não exposta da mesa de concreto. Para 
esta superfície de convecção foi admitido um segundo "Nó extra 1" para o controle da temperatura, sendo admitida para este segundo nó extra uma temperatura constante e igual a $20{ }^{\circ} \mathrm{C}$. Essa estratégia foi utilizada para a simulação da superfície superior da viga, recoberta com manta refratária no ensaio experimental no caso do ensaio da viga 1 . Para o segundo ensaio da viga não foi considerada essa superfície para manter a temperatura constante em $20^{\circ} \mathrm{C}$, já que não foi utilizada manta refratária no segundo ensaio. Para permitir a transferência de calor na interface dos materiais foi utilizado o comando "CPINTF, TEMP, 0,00001", do software [1], acoplando o grau de liberdade "temperatura" para nós sobrepostos. A Figura 8 também mostra a seção discretizada com as superfícies de radiação e convecção admitidas no modelo.

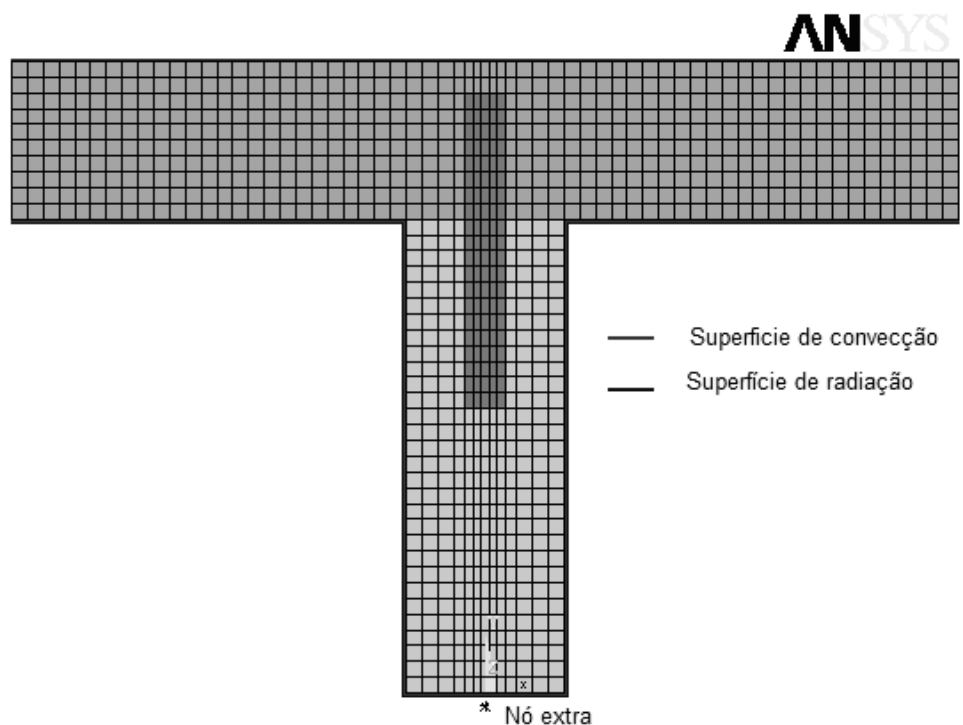

Figura 8: Superfícies (radiação e convecção) aplicadas à seção transversal do modelo numérico para aquecimento segundo curva padronizada [10].

A análise considerada no modelo foi do tipo "térmica transiente". A norma [10] recomenda a curva representada pela Equação (3) como modelo de incêndio padrão.

$\theta_{g}=\theta_{0}+345 \cdot \log .(8 . t+1)$

Onde: $\theta g$ é temperatura dos gases do ambiente em chamas ( $\left.{ }^{\circ} \mathrm{C}\right) ; \theta_{0}$ a temperatura dos gases no momento (tomada $=20^{\circ} \mathrm{C}$ ) e $t$ é tempo (minutos).

\subsubsection{Propriedades térmicas admitidas para o concreto}

A condutividade térmica do concreto, $\lambda$, admitida no modelo numérico, variou com a temperatura $\theta$ dada conforme Equação (4), [12,13].

$$
\lambda(\theta)= \begin{cases}1.748 & \text { If } \theta \in[20 ; 199]^{\circ} \mathrm{C} \\ 1.748-1.246 \times 10^{-3} & \text { If } \theta \in[199 ; 899]^{\circ} \mathrm{C} \\ 0.846 & \text { If } \theta>899^{\circ} \mathrm{C}\end{cases}
$$

$\mathrm{O}$ calor específico do concreto em função da temperatura foi admitido com valor de $1000 \mathrm{~J} / \mathrm{kg} .{ }^{\circ} \mathrm{C}$, conforme [15]. O coeficiente de convecção adotado para o elemento finito de superfície SURF151-convecção que contornou a superfície externa de concreto foi igual a $25 \mathrm{~W} / \mathrm{m}^{2} \mathrm{~K}$, de acordo com [16]. O valor da emissividade adotado para o elemento finito de superfície SURF151-radiação que contornou a superfície inferior da mesa de concreto foi igual a 0,522, de acordo com [13]. A constante de Stefan Boltzmann utilizada no modelo numérico para os efeitos da radiação foi $5,67 \times 10^{-8} \mathrm{~W} / \mathrm{m}^{2} \cdot \mathrm{K}^{4}$, conforme [17]. Este valor foi utilizado nas superfícies de radiação para ambos os materiais, madeira e concreto. Considerou-se concreto com resistência na compressão de $26 \mathrm{MPa}$ e módulo de elasticidade E = $24157 \mathrm{MPa}$, conforme caracterização experimental.

\subsubsection{Propriedades térmicas admitidas para a madeira}

Para a madeira, foi admitido no modelo numérico resistência na compressão de 82,13 MPa e módulo de elasticidade longitudinal de $\mathrm{E}=19727 \mathrm{MPa}$, conforme definido na caracterização experimental. Uma das maio- 
res dificuldades da modelagem numérica de elementos estruturais de madeira em altas temperaturas está relacionada a obtenção de parâmetros térmicos representativos em função da temperatura. A densidade da madeira, assim como o calor específico admitidos no modelo numérico variaram em função do aumento da temperatura, de acordo com [12]. A Figura 9 ilustra as propriedades da madeira em função da temperatura, calibradas para madeiras de Eucalyptus citriodora [12]. O valor do coeficiente de convecção adotado para os elementos do contorno (SURF151-convecção) da alma da madeira foi igual a $13,5 \mathrm{~W} / \mathrm{m}^{2} . \mathrm{K}$, de acordo com [18], e o valor da emissividade adotado para os elementos finitos (SURF151-radiação) que contornaram a superfície da alma de madeira foi igual a 0,6, conforme [19]. A curva de condutividade térmica, Figura 10, foi calibrada para se obter uma relação adequada entre os resultados experimentais e o modelo numérico, [12].

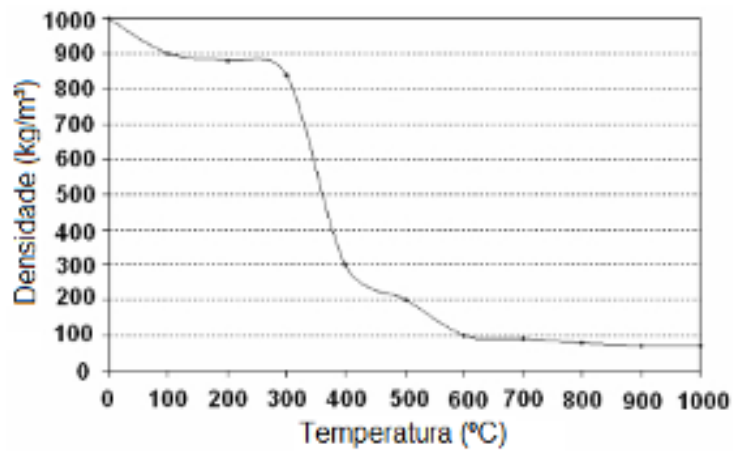

(a)

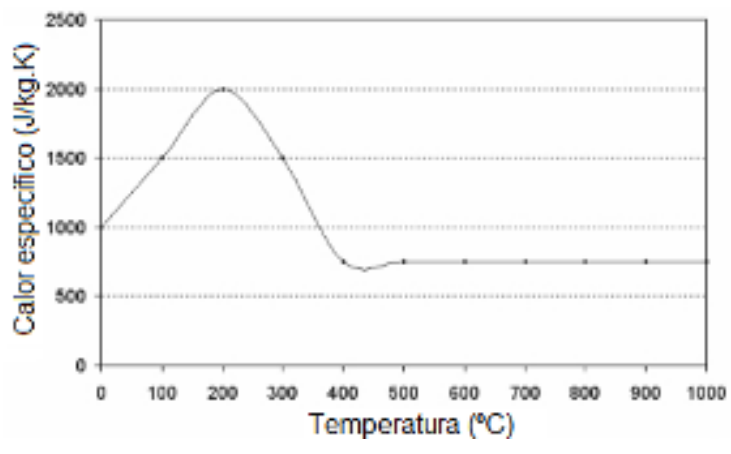

(b)

Figura 9: Propriedades térmicas admitidas para a madeira: (a) Densidade; (b) Calor específico. Fonte: [12].

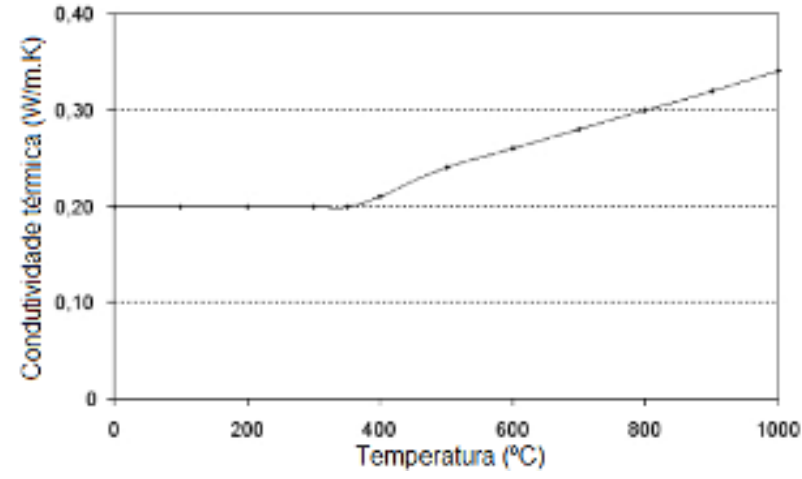

Figura 10: Condutividade térmica da madeira. Fonte: [12]

\subsubsection{Propriedades térmicas do aço dos conectores}

O valor da densidade admitido para o aço foi igual a $7850 \mathrm{~kg} / \mathrm{m}^{3}$. A curva do calor específico do aço em função da temperatura, assim como da condutividade térmica em função da temperatura, dados de acordo com [17] estão apresentadas na Figura 11.
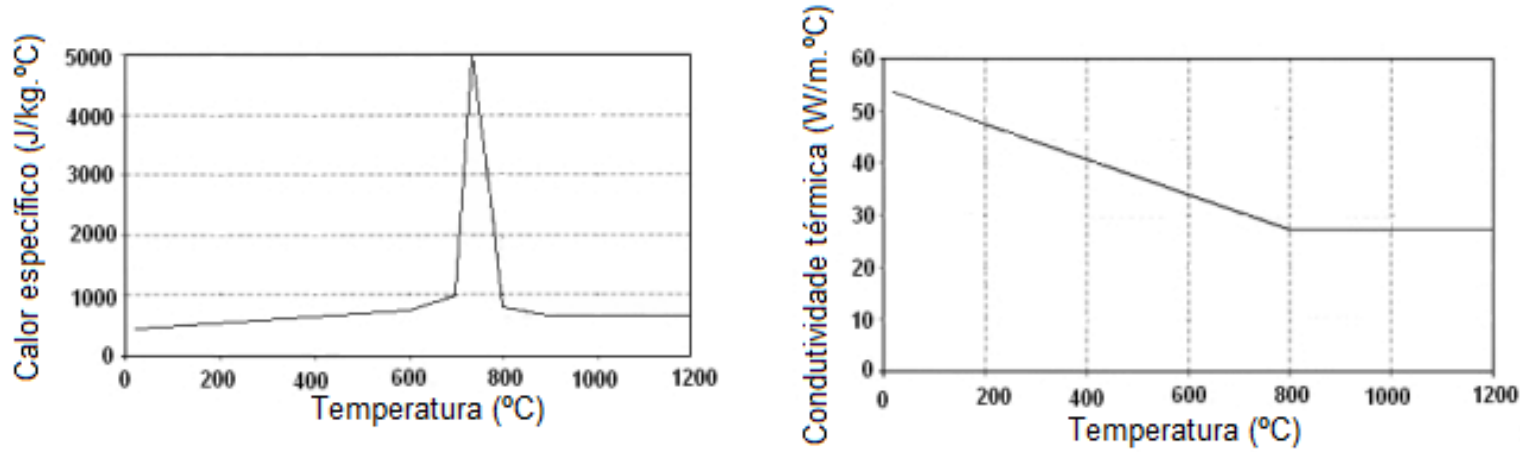

Figura 11: Propriedades térmicas do aço dos conectores: (a) Calor específico; (b) Condutividade térmica. Fonte: [17].

\section{RESULTADOS E DISCUSSÕES}




\subsection{Ensaio Viga 1}

O ensaio realizado com a primeira viga mista teve duração aproximada de 20 minutos e foi interrompido devido a problemas de instabilidade e superaquecimento da viga de aço I posicionada no interior do forno para a aplicação do carregamento nos terços do vão da viga. O isolamento com manta refratária, neste caso, foi deficiente e permitiu assim a entrada de calor, que logo foi percebido pelo sistema de aquisição de dados através de um termopar instalado na viga I. No entanto, foi possível capturar os valores da elevação da temperatura na seção T, no pontos da Figura 3(a). A Figura 12 mostra a comparação das temperaturas para dois pontos da seção transversal T (2 e 5) e, neste caso, são apresentadas as seguintes curvas: ISO 834 - Teórica; Curva Numérica [1]; Curva experimental (leitura dos termopares) e Controle do forno (média de 09 termopares internos para controle da temperatura em todos os pontos do forno.

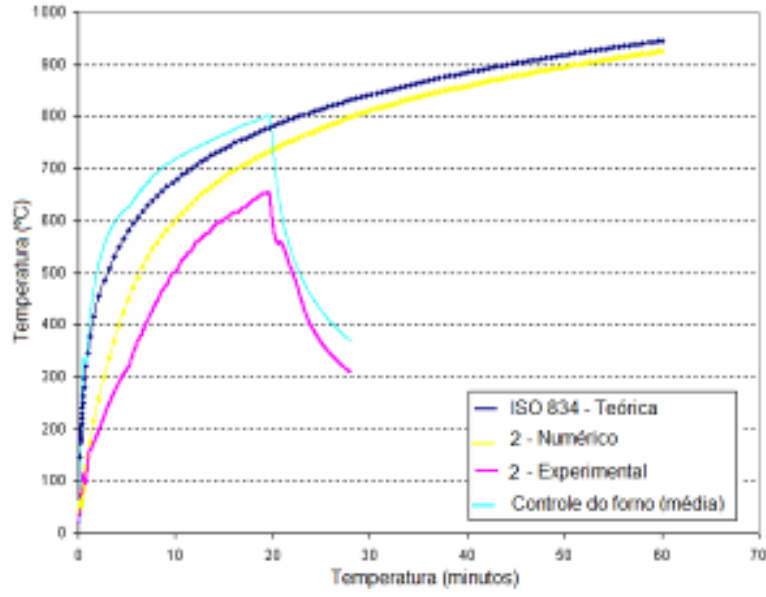

(a)

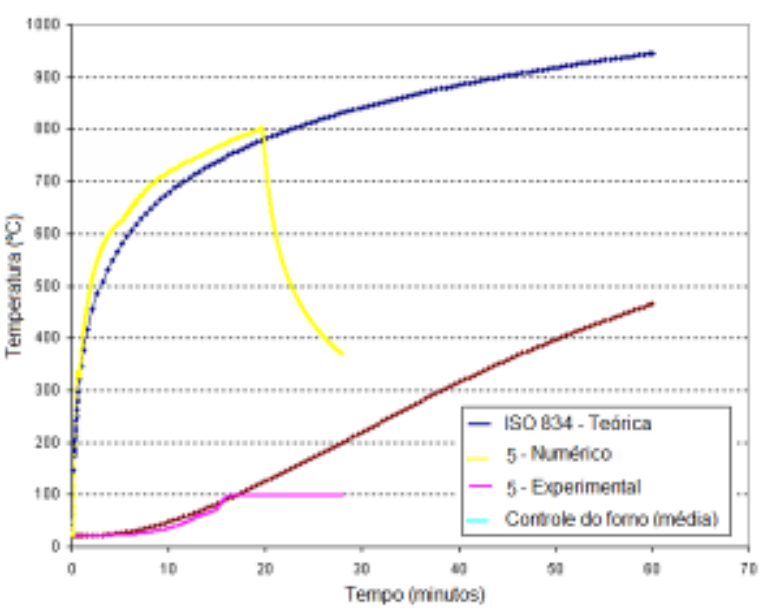

(b)

Figura 12: Comparação entre os resultados numéricos e experimentais obtidos para a seção transversal T: (a) Ponto 2; (b) Ponto 5.

Após a análise da Figura 12, observou-se que, de uma maneira geral, houve uma boa concordância entre os valores numéricos e experimentais de temperatura obtidos para todos os pontos da seção transversal para um tempo de exposição de até 20 minutos ao incêndio padrão. É importante salientar também que os valores de temperatura considerados na seção transversal $\mathrm{T}$ das vigas mistas para comparação foram obtidos numericamente pelo software [1] para os tempos totais de 20 minutos no caso da viga 1 ( $1^{\circ}$ ensaio) e de 30 minutos no caso da viga 2 ( $2^{\circ}$ ensaio).

Os perfis de temperatura para as vigas 1 e 2 são apresentados nas Figuras 13 e 14 para os referidos tempos de exposição ao incêndio padrão [10].

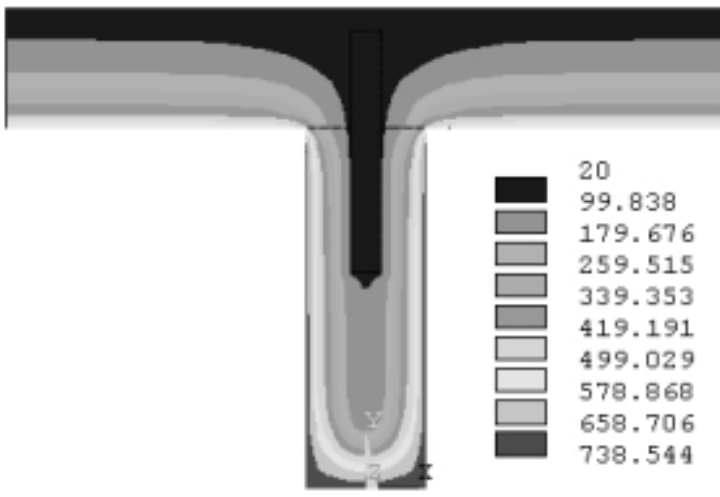

(a)

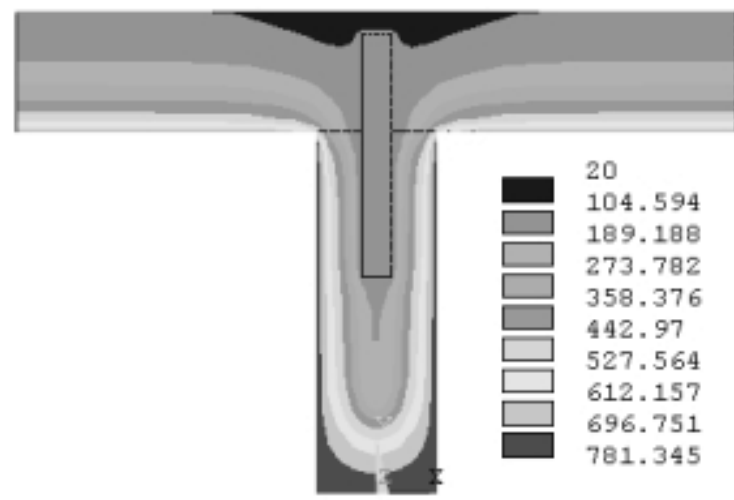

(b)

Figura 13: Campos de temperatura para a viga 1: (a) 15 minutos de exposição ao fogo; (b) 20 minutos de exposição. 
Para a viga 1, para um tempo de 20 minutos de exposição, os resultados numéricos dos perfis de temperatura mostraram que o valor mais elevado de temperatura obtido na seção transversal foi de $781{ }^{\circ} \mathrm{C}$, que ocorreu na superfície inferior da alma de madeira. Para a superfície de inferior da mesa de concreto o valor de temperatura obtido foi de $696{ }^{\circ} \mathrm{C}$. Na região dos conectores da viga 1 a temperatura chegou a $358,37{ }^{\circ} \mathrm{C}$ indicando que a manta refratária teve grande influência na contenção da temperatura.

\subsection{Ensaio Viga 2}

O segundo ensaio experimental, realizado com carregamento distribuído considerado, relativo ao peso próprio da viga mista, ou seja, sem o carregamento nos terços do vão, teve duração aproximada de 35 minutos e foi finalizado devido a flecha da viga mista, neste caso, ter ultrapassado o deslocamento limite normalizado por flexão [10]. O deslocamento vertical no meio do vão, adotado como critério de parada do ensaio, foi igual a 32,5 cm, sendo que o valor da velocidade de ensaio de 14,45 mm/min (taxa de deflexão) deveria ser quantificado após a viga 2 atingir um deslocamento vertical de $17 \mathrm{~cm}$ no meio do vão, conforme [10], o que não foi possível. Em outras palavras, o critério de parada deste segundo ensaio foi o valor da flecha limite no meio do vão da viga.

Vale dizer ainda que a mesma aproximação observada entre os resultados numéricos e experimentais das curvas de temperatura da Figura 12 para os pontos da seção da viga 1 foram também observados para os pontos da segunda viga ensaiada. Esse fato indica que o sistema de ensaio e de aquisição de dados para altas temperaturas do forno horizontal de ensaios da EESC/USP está com funcionamento adequado conforme esperado.

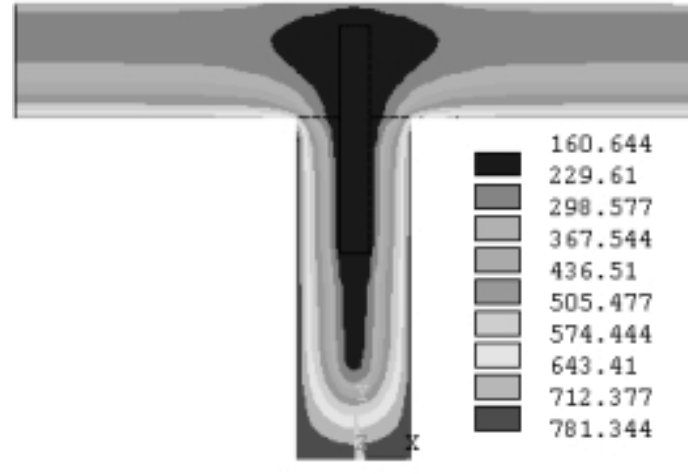

(a)

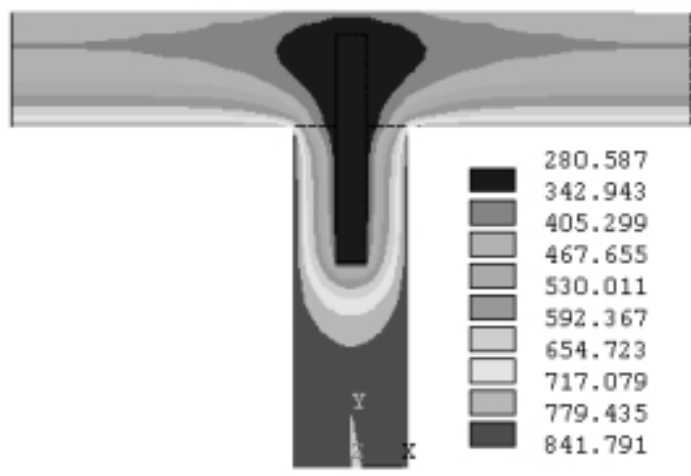

(b)

Figura 14: Campos de temperatura para a viga 2: (a) 20 minutos de exposição ao fogo; (b) 30 minutos de exposição.

Para a viga 2, o maior valor de temperatura obtido na seção para o tempo de exposição de 30 minutos foi de aproximadamente $841,79^{\circ} \mathrm{C}$ para a alma de madeira e na parte inferior da mesa de concreto a temperatura chegou a $779,43{ }^{\circ} \mathrm{C}$. Nas regiões dos conectores a temperatura chegou a $530{ }^{\circ} \mathrm{C}$, valor este maior que o encontrado para a viga 1 provavelmente devido a não utilização da manta refratária na superfície superior da mesa de concreto, no caso da viga 2.

\subsection{Situação da viga após o ensaio em altas temperaturas}

Em um incêndio padronizado o carvão formado pela carbonização da madeira sofre craquelamento, permitindo a penetração rápida dos gases quentes pelas fendas formadas na madeira. A Figura 15(a) mostra os detalhes da viga mista após o ensaio em altas temperaturas e a Figura 15(b) os detalhes da peça de madeira com sinais de craquelamento. 


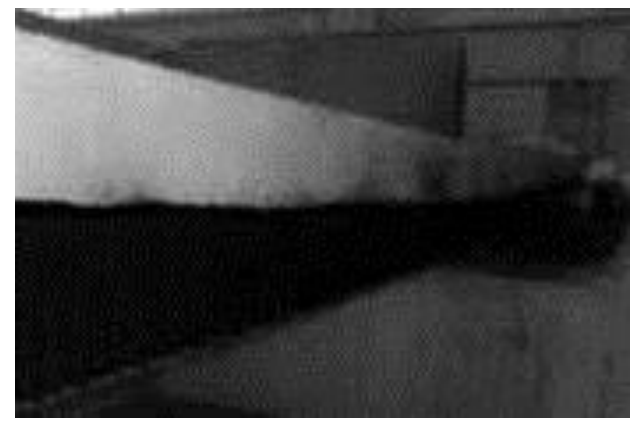

(a)

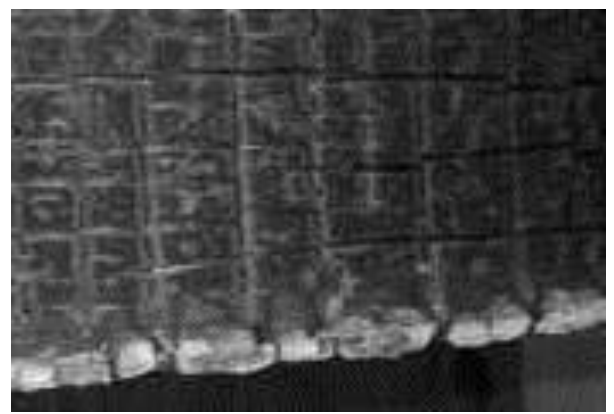

(b)

Figura 15: Viga mista após ensaio no forno: (a) Viga 1; (b) Craquelamento da peça de madeira.

\section{CONCLUSÕES}

As vigas mistas 1 e 2, ensaiadas em altas temperaturas no forno horizontal construído na EESC/USP, resistiram, respectivamente, a 20 e 30 minutos, de acordo com aquecimento padronizado pela norma [10] permitindo assim a verificação de seus comportamentos resistentes na flexão. Os termopares instalados na seção transversal $\mathrm{T}$ das vigas forneceram adequadamente os valores das temperaturas experimentais nos pontos pré-determinados da seção transversal mista T. Os valores dos perfis de temperatura obtidos numericamente através do software ANSYS [1] concordaram com os valores obtidos experimentalmente a partir da utilização do forno de ensaios. Além disso, as curvas de temperatura em função do tempo obtidas numericamente pelo software [1] para os pontos pré-definidos da seção transversal concordaram com as curvas experimentais para os tempos analisados de 20 e 30 minutos, referentes as vigas 1 e 2, respectivamente. As curvas de temperatura experimental versus tempo de incêndio referentes ao controle interno do forno também concordaram com a curva teórica proposta pela norma [10] considerada neste estudo, mostrando assim a boa calibração do forno de ensaios. O modelo numérico apresentado neste trabalho possibilitou a obtenção da temperatura na seção $\mathrm{T}$ para o elemento concreto, de madeira e, principalmente, para a região dos conectores metálicos com razoável aproximação. Por fim, tem-se que o presente estudo numérico-experimental envolvendo o sistema misto de madeira e concreto com sistema de conexão metálico, em altas temperaturas, foi o primeiro estudo realizado no Brasil e, portanto, servirá como ponto de partida para estudos futuros envolvendo a instrumentação e análise destes elementos em altas temperaturas. Por outro lado, vale mencionar também, que os ensaios apresentados, neste trabalho, foram os primeiros realizados no Brasil em forno horizontal com carregamento aplicado, tendo em vista a calibração do referido equipamento de ensaios.

\section{AGRADECIMENTOS}

Os autores deste trabalho agradecem à Fundação de Amparo à Pesquisa do Estado de São Paulo - FAPESP pelo apoio financeiro desta pesquisa. O financiamento refere-se ao processo de número 08/06726-5, relativo a bolsa de Pós-Doutorado.

\section{BIBLIOGRÁFIA}

[1] ANSYS INC. “Ansys Release 10.0”, Documentation. 2005.

[2] O’NEILL, J.W., The fire performance of Timber-Concrete composite floor, Thesis of D.Sc., Department of Civil and Natural Resources Engineering/University of Canterbury, Christchurch, New Zealand, 2009.

[3] FRANGI, A., KNOBLOCH, M., FONTANA, M., "Fire Design of Timber-Concrete Composite Slabs with Screwed Connections”, Journal of structural engineering, v. 136, n. 2, pp. 219 - 228, Feb. 2010.

[4] KODUR, V., STEIN, D., FIKE, R., et al., "Comparison of Fire Resistance of Traditional Solid Joist Lumber and Engineered I-joists", In: Proceedings of $7^{\text {th }}$ International Conference on Structures in Fire: SiF, pp. 417-426, Zurich, Jun. 2012.

[5] PINTO, E.M., RIGOBELlO, R. MUNAIAR NETO, J., CALIL JUNIOR, C. "Theoretical and experimental study of the thermal degradation of Eucalyptus timber", Forest Products Journal, v. 58, pp. 85-89, Abr. 2008. 
[6] KIRCHHOF, L., MUNAIAR NETO, J., MALITE, M., "Simulacion numérica de vigas mixtas de aceroconcreto en temperatura ambiente y en situación de incêndio", Revista Sul-Americana de Engenharia Estrutural, v. 2, n.2, pp. 87-102, Maio/Ago. 2005.

[7] RIGOBELLO, R., MUNAIAR NETO, J., "Análise numérica de seções transversais e de elementos estruturais de aço e mistos de aço e concreto em situação de incêndio", Cadernos de Engenharia de Estruturas (Online), v. 10, pp. 96-116, 2008.

[8] MORENO JUNIOR, A.L., MOLINA, J.C., CALIL JUNIOR, C., "Considerações sobre a concepção do primeiro forno brasileiro para avaliação de lajes e vigas, carregadas, em situação de incêndio", REM: Revista Escola de Minas, v. 66, n. 1, pp. 25-33, Jan/Mar. 2013.

[9] KODUR, V.K.R., FIKE, R., "Guidelines for Improving the Standard Fire Resistance Test Specification", Journal of ASTM International, v. 6, n. 7, pp. 1-16, July. 2009.

[10] INTERNATIONAL STANDARD ORGANIZATION. ISO 834:1999: Fire resistance tests - Elements of building construction, Part 1 - General requirements. Switzerland, 1999.

[11] PINTO, E.M., Determinação de um modelo de taxa de carbonização transversal a grã para o eucalyptus citriodora e eucalyptus grandis,Tese de D.Sc., EESC/USP, São Carlos, SP, Brasil 2005.

[12] REGOBELLO, R., Análise numérica de seções transversais e de elementos estruturais de aço e mistos de aço e concreto em situação de incêndio, Dissertação de M.Sc., EESC/USP, São Carlos, SP, Brasil, 2007.

[13] SILVA, J.C., Comportamento de estruturas de aço e estruturas compostas em situação de incêndio: Modelagem e aplicações, Dissertação de M.Sc., PPEC/UFES, Vitória, ES, Brasil, 2002.

[14] KIRCHHOF, L. D., MUNAIAR NETO, J., "Uma contribuição ao estudo de vigas mistas de madeira e concreto simplesmente apoiadas em temperatura ambiente e em situação de incêndio", Caderno de Engenharia de Estruturas, v.7, n. 25, pp. 1-31, 2005.

[15] EUROPEAN COMMITTEE FOR STANDARDISATION. EUROCODE 4: Design of composite steel and concrete structures, Part 1.2: General Rules - Structural Fire Design, Brussels, 2002.

[16] ASSOCIAÇÃO BRASILEIRA DE NORMAS TÉCNICAS. NBR 14323: Dimensionamento de estruturas de aço de edifícios em situação de incêndio. Rio de Janeiro, 1999.

[17] EUROPEAN COMMITTEE FOR STANDARDISATION. EUROCODE 3: Design of steel structures: Structural fire design, Part 1.2: General rules, Brussels, 2002.

[18] POON, L., ENGLAND, J.P., Literature review on the contribution of fire resistant timber construction to heat release rate. Warrington Fire Research. Victoria, 2003.

[19] JONES, B.H., Performance of gypsum plasterboard assemblies exposed to real building fires, Research report for the degree of Master of Engineering in Fire Engineering, Department of Civil Engineering/University of Canterbury, Christchurch, New Zealand, 2001. 